\title{
Caracterização físico-química e sensorial de frozen iogurte de kiwi enriquecido com prebiótico
}

\author{
Phisico-chemical and sensory characterization of frozen kiwi yogurt enriched with prebiotic \\ Caracterización físico-química y sensorial del yogur de kiwi helado enriquecido con prebióticos
}

Recebido: 10/06/2021 | Revisado: 17/06/2021 | Aceito: 20/06/2021 | Publicado: 04/07/2021

Natiéli Piovesan

ORCID: https://orcid.org/0000-0001-6920-1722 Instituto Federal de Educação, Ciência e Tecnologia do Rio Grande do Norte, Brasil

E-mail: natieli.piovesan@ifrn.edu.br

Mirla Fontes de Araújo Borges

ORCID: https://orcid.org/0000-0002-1593-0783 Instituto Federal de Educação, Ciência e Tecnologia do Rio Grande do Norte, Brasil E-mail: mirlafontes15@gmail.com

Joyce Moura de Queiroz ORCID: https://orcid.org/0000-0003-4431-0337 Instituto Federal de Educação, Ciência e Tecnologia do Rio Grande do Norte, Brasil

E-mail: joycemouraq@outlook.com

Rosane Liége Alves de Souza

ORCID: https://orcid.org/0000-0002-2269-1442 Instituto Federal de Educação, Ciência e Tecnologia do Rio Grande do Norte, Brasil E-mail: rosaneliege@yahoo.com.br

Emanuel Neto Alves de Oliveira ORCID: https://orcid.org/0000-0002-7000-8126 Instituto Federal de Educação, Ciência e Tecnologia do Rio Grande do Norte, Brasil E-mail: Emanuel.oliveira16@gmail.com

Gleison Silva Oliveira

ORCID: https://orcid.org/0000-0002-6569-1493 Instituto Federal de Educação, Ciência e Tecnologia do Rio Grande do Norte, Brasil E-mail: gleison.silva@ifrn.edu.br

Adalva Lopes Machado

ORCID: https://orcid.org/0000-0001-9993-1646 Instituto Federal de Educação, Ciência e Tecnologia do Rio Grande do Norte, Brasil E-mail: adalva.machado@ifrn.edu.br

Pâmara Virna Carlos de Oliveira ORCID: https://orcid.org/0000-0003-4380-6023 Universidade Federal do Rio Grande do Norte, Brasil E-mail: pamaravirna@ outlook.com

Palloma Vitória Carlos de Oliveira ORCID: https://orcid.org/0000-0002-8855-6008

Universidade Federal Rural do Semi-Árido, Brasil E-mail: pallomavictoria@hotmail.com.br

\begin{abstract}
Resumo
O frozen iogurte chama atenção por aproveitar os nutrientes do iogurte e refrescância do sorvete, além de poder ser enriquecido com frutas que garantam o sabor, vitaminas e atividade antioxidante, como também a adição de prebióticos, permitindo maior funcionalidade e os respectivos benefícios à saúde. Portanto, o objetivo deste trabalho foi elaborar frozen iogurte sabor kiwi enriquecido com frutooligossacarídeo. Para isso, foram desenvolvidas a formulação padrão (sem prebiótico) F1 e F2 com adição de 5 e 10\% de prebiótico, respectivamente. De acordo com as análises físico-químicas, os parâmetros de $\mathrm{pH}$, umidade, cinzas e proteínas foram os que sofreram diferenças significativas. Os lipídios, apesar de não terem sido influenciados pela quantidade do prebiótico, ficaram dentro da legislação. $\mathrm{Na}$ análise sensorial, as amostras obtiveram média acima de 7 para todos os atributos sensoriais, correspondendo a "gostei moderadamente" na escala hedônica, demonstrando que a adição do frutooligossacarídeo não influenciou negativamente nas características do produto, podendo ser alternativa saborosa e viável para o aproveitamento da funcionalidade dos frutooligossacrídeos.
\end{abstract}

Palavras-chave: Aceitabilidade; Sorvete; Bactérias intestinais benéficas.

\section{Abstract}

Frozen yogurt draws attention by taking advantage of the nutrients of yogurt and refreshing ice cream, in addition to being enriched with fruits that guarantee flavor, vitamins and antioxidant activity, as well as the addition of prebiotics, 
allowing greater functionality and the respective health benefits. Therefore, the objective of this work was to elaborate frozen kiwi flavored yogurt enriched with fructooligosaccharide. For this, the standard formulation (without prebiotic) F1 and F2 was developed with the addition of 5 and $10 \%$ of prebiotic, respectively. According to the physicalchemical analyzes, the parameters of $\mathrm{pH}$, humidity, ash and proteins were the ones that suffered significant differences. The lipids, although not influenced by the amount of the prebiotic, were within the legislation. In the sensory analysis, the samples obtained an average above 7 for all sensory attributes, corresponding to "I liked it moderately" on the hedonic scale, demonstrating that the addition of the fructooligosaccharide did not negatively influence the characteristics of the product, being able to be a tasty and viable alternative for the use. of the functionality of fructooligosaccharide.

Keywords: Acceptability; Ice cream; Beneficial intestinal bactéria.

\section{Resumen}

El yogur helado llama la atención al aprovechar los nutrientes del yogur y del helado refrescante, además de estar enriquecido con frutas que garantizan el sabor, vitaminas y actividad antioxidante, así como la adición de prebióticos, permitiendo una mayor funcionalidad y los respectivos beneficios para la salud. Por tanto, el objetivo de este trabajo fue elaborar yogur helado con sabor a kiwi enriquecido con fructooligosacárido. Para ello, se desarrolló la formulación estándar (sin prebiótico) F1 y F2 con la adición de 5 y $10 \%$ de prebiótico, respectivamente. Según los análisis físico-químicos, los parámetros de $\mathrm{pH}$, humedad, cenizas y proteínas fueron los que sufrieron diferencias significativas. Los lípidos, a pesar de no estar influenciados por la cantidad de prebiótico, estaban dentro de la legislación. En el análisis sensorial, las muestras obtuvieron una media superior a 7 para todos los atributos sensoriales, correspondiente a "Me gustó moderadamente" en la escala hedónica, demostrando que la adición del fructooligosacárido no influyó negativamente en las características del producto, siendo un sabroso y alternativa viable de la funcionalidad de los fructooligosacridos.

Palabras clave: Aceptabilidad; Helado; Bacterias intestinales beneficiosas.

\section{Introdução}

Segundo a Agência Nacional de Vigilância Sanitária, sorvete ou gelado comestível é "um produto alimentício obtido a partir de uma emulsão de gordura e proteínas, com ou sem adição de outros ingredientes e substâncias, ou de uma mistura de água, açúcares e outros ingredientes e substâncias que tenham sido submetidas ao congelamento, em condições tais que garantam a conservação do produto no estado congelado ou parcialmente congelado, durante a armazenagem, o transporte e a entrega ao consumo". Dentro dessa categoria, encontram-se as sobremesas lácteas, constituídas de materiais lácteos e não lácteos, com variedade de opções e proporções adequadas, congeladas ou não, aeradas e cremosas, como por exemplo, os sorvetes, mousses, frozen iogurte e flans (Juliano et al., 2014).

O frozen iogurte é uma sobremesa fermentada e congelada que associa o valor nutricional do iogurte com o sabor refrescante do sorvete, possui baixo teor de gordura e lactose (Goff, 2011). No Brasil e no mundo, já ganhou espaço com conceitos que envolvem saúde, sabor diferenciado e leveza, atingindo consumidores desde bebês de colo a mais idosos que consomem no lanche ou até mesmo substituindo refeições (Finamac, 2012).

O "frozen iogurte" ou "sorvete de iogurte", como é mais conhecido no Brasil, é um produto obtido basicamente com leite, submetidos à fermentação láctea através da ação de Streptococcus thermophilus e Lactobacillus bulgaricus, ou a partir de iogurte com ou sem adição de outras substâncias alimentícias, sendo posteriormente aerado e congelado (Anvisa, 2000), além de receber sabores únicos (Monteiro et al., 2015) e exóticos (Araújo, 2011). O sabor pode vir de frutas como kiwi, que apesar de se tratar de fruta ácida e de alto custo, mas contém vitamina $\mathrm{C}$ e desempenha comprovada atividade antioxidante (Gomes, 2012; Soquetta, 2015; Silva et al., 2016).

E assim, o frozen iogurte pode tornar-se alternativa saudável ao sorvete por sua composição nutricional e seu conteúdo em proteínas, cálcio e vitaminas (Pereira et al., 2012; An \& Jiang, 2016). Podem facilmente receber suplementação de nutrientes, inclusão de nutracêuticos, alegação de propriedades funcionais e ter baixo ou nenhum conteúdo de gordura (Alves et al., 2009; Isik et al., 2011; Fernandes et al., 2017).

Nesse sentido, cresce a oferta em alimentos funcionais, e consequentemente o desenvolvimento de produtos com estas características. $\mathrm{Na}$ área de sobremesas lácteas, não é diferente. E pesquisas vem sendo desenvolvidas tentando deixar estes 
alimentos mais saudáveis. Vem daí o interesse na adição dos frutooligossacarídeos (FOS), prebióticos cuja estrutura química não permite a hidrólise por enzimas digestivas e que por isso poderia ser adicionado nos alimentos como substituto de açúcar, apresentando maior solubilidade e cerca de um terço do poder adoçante da sacarose, sem cristalizar, sem precipitar (Zacarchenco et al., 2013), porém sem aumentar a absorção deste macronutriente pelo organismo. Desta forma, diminuiria os riscos à saúde, como aqueles associados com diabetes e obesidade (Chen \& Karboune, 2019).

A legislação prevê que categoria de "Alimentos com Alegações de Propriedade Funcional e ou de Saúde", sejam aqueles cuja porção diária forneça no mínimo $3 \mathrm{~g}$ de inulina ou FOS, se o alimento for sólido, ou 1,5g, se o alimento for líquido, e podem apresentar a alegação de "contribuir para o equilíbrio da flora intestinal" (Brasil, 2008). Em geral, a alimentação com FOS, fornece produtos do metabolismo como ácidos graxos de cadeia curta, que podem ser utilizados por bifidobactérias e lactobacilos, consideradas como probióticas (Al-Sherajia et al., 2013). Ainda à sua ingestão atribui-se diversos efeitos benéficos à saúde, como por exemplo, prevenção de cáries dentárias, redução nos níveis séricos de colesterol total e lipídeos (Passos \& Park, 2003).

Portanto, o objetivo do presente trabalho foi elaborar frozen iogurte de kiwi enriquecido com frutooligossacarídeo e avaliar as características físico-químicas e sensoriais.

\section{Metodologia}

O frozen iogurte de kiwi foi elaborado no Laboratório de Processamento de Leite e Derivados, enquanto que a análise sensorial ocorreu no Laboratório de Análise Sensorial e a caracterização físico-química no Laboratório de Análises FísicoQuímica de Alimentos do Instituto Federal de Ciência e Tecnologia do Rio Grande do Norte Campus Pau dos Ferros-RN.

Os ingredientes: leite em pó, liga neutra, açúcar, emulsificante (Du porto), glicose de milho, leite integral UHT, cultura láctea, prebiótico frutooligossacarídeo, corante verde (Selecta) e kiwi, foram adquiridos no comércio local das cidades de José da Penha-RN e Pau dos Ferros-RN.

\subsection{Elaboração do frozen iogurte de kiwi}

Inicialmente elaborou-se o iogurte a partir da mistura de leite integral UHT, açúcar (10\%) e leite em pó (6\%), que foi pasteurizada a $90^{\circ} \mathrm{C}$ durante cinco minutos. A cultura láctea $(2 \%)$ foi então adicionada à $42^{\circ} \mathrm{C}$ e a incubação ocorreu na mesma temperatura em estufa B.O.D por aproximadamente 6 horas. Em seguida, armazenou-se o iogurte sob refrigeração $\left(7^{\circ} \mathrm{C}\right)$ para posterior preparação do frozen iogurte.

Para obtenção do concentrado de kiwi, os frutos foram higienizados, descascados e processados em liquidificador industrial. Após isso, misturou-se a polpa com $10 \%$ de açúcar, aquecendo-se em seguida a $100^{\circ} \mathrm{C}$, por cinco minutos. Com o iogurte e o concentrado prontos, foram elaboradas três formulações de frozen iogurte, sendo a padrão sem adição de prebiótico, F1 e F2 com 5\% e 10\% de frutooligossacarídeo, respectivamente, conforme descrição dos ingredientes na Tabela 1.

Definida as formulações, o iogurte, o açúcar, a liga neutra, a glicose de milho e o emulsificante foram misturados em liquidificador por 5 min e a mistura armazenada a $-12^{\circ} \mathrm{C}$ durante $24 \mathrm{~h}$, para que ocorresse a maturação. Após este período, a mistura congelada foi submetida ao batimento, para deixá-la aerada. Acrescentou-se então, o concentrado de kiwi, o prebiótico e o corante, seguida de homogeneização durante $15 \mathrm{~min}$. O frozen foi congelado durante $24 \mathrm{~h}$ em potes de polietileno higienizados. 
Tabela 1. Proporção dos ingredientes de cada formulação do frozen iogurte.

\begin{tabular}{|c|c|c|c|}
\hline Ingredientes & Padrão & F2 & F3 \\
\hline Iogurte & $56 \%$ & $51 \%$ & $46 \%$ \\
\hline Açúcar & $12 \%$ & $12 \%$ & $12 \%$ \\
\hline Liga Neutra & $1 \%$ & $1 \%$ & $1 \%$ \\
\hline Glucose de milho & $1 \%$ & $1 \%$ & $1 \%$ \\
\hline Emulsificante & $1 \%$ & $1 \%$ & $1 \%$ \\
\hline Leite em pó & $4 \%$ & $4 \%$ & $4 \%$ \\
\hline Frutooligossacarídeo & 0 & $5 \%$ & $10 \%$ \\
\hline Concentrado de kiwi & $24,5 \%$ & $24,5 \%$ & $24,5 \%$ \\
\hline Corante & $0,5 \%$ & $0,5 \%$ & $0,5 \%$ \\
\hline
\end{tabular}

Fonte: Autores.

\subsection{Análises físico-químicas}

As análises físico-químicas foram realizadas em triplicata para os seguintes parâmetros: umidade (estufa a $105^{\circ} \mathrm{C}$ por 24 horas), cinzas (em mufla, a $550^{\circ} \mathrm{C}$ por 6 horas), proteínas adotando o fator de conversão de 6,28 para leite e derivados lácteos, através método micro Kjeldahl (Aoac, 2010), pH (por potenciometria em pHmetro digital). Executou-se a análise de atividade de água em um determinador de atividade de água e açúcares totais de acordo com o método titulométrico de oxirredução descritos pelo Mapa, 2005 e Instituto Adolfo Lutz (2008). Realizou-se somente a análise de lipídeos em duplicata, pelo método de extração a fio segundo Folch et al. (1957).

\subsection{Análise sensorial}

Foi aplicado teste de aceitação por escala hedônica a 80 provadores não treinados, de ambos os sexos, compostos por alunos e servidores do IFRN - Campus Pau dos ferros, na faixa etária de 14 a 39 anos, baseando-se na metodologia de Dutcosky (2013). As amostras de $30 \mathrm{~mL}$ foram servidas em copos brancos descartáveis codificados com números aleatórios de três dígitos e apresentadas aos julgadores, que foram orientados a prová-las da esquerda para a direita, bebendo água entre as amostras para a limpeza do palato. Foram avaliados os parâmetros cor, aroma, textura, aparência e sabor de cada formulação, através da seguinte escala: 1 (desgostei extremamente); 2 (desgostei muito); 3 (desgostei moderadamente); 4 (desgostei ligeiramente); 5 (indiferente); 6 (gostei ligeiramente); 7 (gostei moderadamente); 8 (gostei muito); e 9 (gostei extremamente). Para a intenção de compra foram atribuídas as notas 1 (certamente não compraria); 2 (provavelmente não compraria); 3 (provavelmente compraria); e 4 (certamente compraria).

\subsection{Análise Estatística}

Para a análise estatística dos dados, foi aplicado o delineamento inteiramente casualizado, com o teste de médias pelo teste Tukey a 5\% de probabilidade, através do software Statistica 7.0 (Statsoft, 2004).

\section{Resultados e Discussão}

\subsection{Análises físico-químicas}

A caracterização físico-química das amostras do frozen iogurte estão apresentadas na Tabela 2. 
Tabela 2. Caracterização físico-química das formulações de frozen iogurte.

\begin{tabular}{cccc}
\hline Análises & Padrão & F1 & F2 \\
\hline pH & $4,49 \pm 0,06^{\mathrm{a}}$ & $4,40 \pm 0,01^{\mathrm{b}}$ & $4,44 \pm 0,02^{\mathrm{ab}}$ \\
Atividade de água (aw) & $0,89 \pm 0,01^{\mathrm{a}}$ & $0,89 \pm 0,01^{\mathrm{a}}$ & $0,89 \pm 0,01^{\mathrm{a}}$ \\
Umidade (\%) & $58,25 \pm 0,64^{\mathrm{a}}$ & $56,26 \pm 0,40^{\mathrm{b}}$ & $53,23 \pm 0,65^{\mathrm{c}}$ \\
Cinzas (\%) & $0,96 \pm 0,05^{\mathrm{a}}$ & $0,89 \pm 0,02^{\mathrm{ab}}$ & $0,84 \pm 0,05^{\mathrm{b}}$ \\
Lipídeos (\%) & $2,73 \pm 0,38^{\mathrm{a}}$ & $3,37 \pm 0,31^{\mathrm{a}}$ & $3,51 \pm 0,43^{\mathrm{a}}$ \\
Proteínas (\%) & $4,40 \pm 0,18^{\mathrm{a}}$ & $3,90 \pm 0,06^{\mathrm{b}}$ & $3,47 \pm 0,03^{\mathrm{c}}$ \\
Açúcares Totais - AT $(\%)$ & $7,44 \pm 0,03^{\mathrm{a}}$ & $7,19 \pm 0,10^{\mathrm{b}}$ & $7,53 \pm 0,08^{\mathrm{a}}$ \\
\hline
\end{tabular}

*Letras idênticas em uma mesma linha indicam resultados estaticamente iguais pelo Teste de Tukey ( $<<0,05)$.

Fonte: Autores.

Para os resultados de $\mathrm{pH}$, nota-se diferença estatística entre as amostras padrão e F1, observando-se pequena redução de pH nas amostras adicionadas de prebiótico em relação à amostra padrão. No entanto, de forma geral, todas as formulações foram caracterizadas como ácidas. Para Pereira et al. (2012), é possível controlar o pH do frozen, já que este é uma sobremesa láctea fermentada e congelada que combina as características físicas do sorvete com as propriedades sensoriais e nutricionais do leite fermentado, e por isso pode-se estabelecer o ponto final de fermentação. Além disso, tanto a formulação padrão, como F1 e F2 seguem o comportamento ácido do kiwi, a fruta que confere o sabor. Conforme Gomes et al. (2012), o pH dessa fruta in natura varia de 2,9 a 3,2. Nogueira et al. (2018), formularam frozen iogurte com culturas probióticas, mas também associaram a alta acidez à fruta saborizante, que neste caso foi a polpa de laranjinha de pacu.

Os valores de atividade de água não apresentaram diferença significativa. Comportamento semelhante foi encontrado por Ribeiro (2012), em seu trabalho de sobremesa com soja aerada potencialmente simbiótica, que obteve valores de aw de 0,96-0,97, sem apresentar variações consideráveis.

Quanto a umidade, observou-se diferença estatística entre as três formulações, havendo redução do valor da umidade à medida que aumentou-se a proporção de frutooligossacarídeo. Os frutooligossacarídeos apresentam cadeias curtas e são muito higroscópicos. Sua capacidade de retenção de água é superior à da sacarose e não participa da reação de Maillard, por ser um carboidrato redutor (Multon, 2007). Em sobremesas lácteas, como no sorvete de iogurte simbiótico de leite de búfala enriquecido com polpa de açaí elaborado por Monteiro et al. (2015), mas sem adição de prebióticos, foram encontrados percentuais maiores $(72,65$ e $74,46 \%)$.

Em relação à análise de cinzas, a amostra padrão diferiu-se estatisticamente da F2, observando um decréscimo do teor de cinzas à medida que se acrescentou o frutooligossacarídeo. Costa et al. (2019), perceberam que dentre iogurtes gregos adicionados de prebiótico, o menor percentual de cinzas foi encontrado na formulação contendo FOS. No entanto, observando a amostra padrão e F1, fica claro que a adição de FOS não interferiu significativamente no conteúdo deste parâmetro.

Atualmente a legislação brasileira não dispõe de padrões para frozen iogurte especificamente, nesse caso, adota-se a Resolução n ${ }^{\circ}$ 266, de 22 de setembro de 2005 (BRASIL, 2005), que aprova o regulamento técnico para fixação de identidade e qualidade de gelados comestíveis, preparados, pós para o preparo e bases para gelados comestíveis, para efeitos de comparação. Segundo a RDC, os valores mínimos estabelecidos para proteínas e gorduras são de 2,5\% para ambos. Os resultados de lipídeos permaneceram entre o intervalo de 2,73 e 3,51\%, dentro do exigido pela legislação, mas para as três formulações não houve diferença significativa, podendo ser justificado pela utilização das mesmas proporções do emulsificante 
nas três formulações.

O conteúdo proteico também atendeu à especificação, e o valor obtido em todos os testes, está acima do mínimo recomendado na legislação $(2,5 \%)$. As três amostras diferiram-se estatisticamente, sendo que a amostra padrão, com maior quantidade de iogurte apresentou valor levemente superior. Aparentemente, o teor de proteína está mais relacionado com a fonte láctea utilizada. Alves et al. (2009), desenvolveram frozen iogurte de leite de cabra com adição de cultura probiótica e prebiótica, e o resultado obtido para proteínas foi de 3\%. Dalla (2008), para frozen iogurte à base de leite de vaca integral contendo prebióticos e probióticos obtiveram 3,18-3,69\% para proteína. Em contrapartida, Abreu et al. (2018), desenvolvendo frozen iogurte a base de leite em pó de ovelha e chegaram a percentuais maiores do que 5\% para proteína.

No conteúdo de açúcares totais somente a amostra padrão diferiu de F2. FOS podem ser usados devido suas propriedades nutricionais e/ou tecnológicas, como substituto de macronutrientes, principalmente gordura e açúcar (Ishwarya \& Prabhasankar, 2013). Como substituto de açúcar, apresentam maior solubilidade e cerca de um terço do poder adoçante da sacarose, não cristalizam, não precipitam, não deixam sensação de secura ou areia na boca (Zacarchenco et al., 2013). Assim no trabalho realizado em questão, os frutooligossacarídeos podem ter contribuído para evitar adição excessiva de açúcar. Souza et al. (2017), obteve valores de açúcares totais que correspondiam a 12,76\% do frozen iogurte sabor tamarindo, neste caso, a fruta mais ácida parece ter exigido conteúdo maior de açúcar durante a formulação.

\subsection{Análise sensorial}

Dos provadores que realizaram a análise sensorial, $48,9 \%$ correspondiam a pessoas do sexo masculino e $51,1 \%$ pertenciam ao sexo feminino. Entre esses mesmos consumidores, 65,5\% estava na faixa etária de 13 aos 17 anos, 30\% tinha entre 18 e 20 anos e 4,5\% eram maiores de 20 anos. Esse cenário é explicado devido a análise ter ocorrido em instituição de ensino médio, onde a quantidade de jovens é predominante.

Em relação a frequência de consumo do frozen iogurte, obteve-se que 65,5\% nunca haviam consumido a sobremesa láctea, 24,5\% disseram consumir ocasionalmente, $7,78 \%$ responderam moderadamente e apenas 2,22\% frequentemente. Segundo a Associação Brasileira das Indústrias de Sorvetes (Abis, 2011) o consumo per capta de sorvetes no Brasil é de 5,77 litros/ano. Mesmo sendo um país tropical, o consumo brasileiro é menor que o consumo de alguns países nórdicos e frios como Suécia, Dinamarca e Finlândia, que apresentam taxas de consumo de: 14,20; 13,90; e 9,20 litros/ano, respectivamente. Mesma tendência pode ser observada para o consumo de frozen.

No que tange o consumo da fruta, 38,9\% disseram que nunca consumiram kiwi, 28,9\% responderam ocasionalmente, $24,4 \%$ responderam que consomem a fruta moderadamente e apenas 7,78\% consomem frequentemente.

Em relação a análise sensorial por escala hedônica, assim como a intenção de compra do produto, os resultados podem ser visualizados na Tabela 3.

Tabela 3. Análise sensorial e intenção de compra do frozen iogurte de kiwi enriquecido com prebiótico.

\begin{tabular}{cccc}
\hline Atributos sensoriais & Padrão & F1 & F2 \\
\hline Aparência & $7,57 \pm 1,54^{\mathrm{a}^{*}}$ & $7,52 \pm 1,34^{\mathrm{a}}$ & $7,58 \pm 1,42^{\mathrm{a}}$ \\
Aroma & $7,29 \pm 1,57^{\mathrm{a}}$ & $7,37 \pm 1,57^{\mathrm{a}}$ & $7,30 \pm 1,68^{\mathrm{a}}$ \\
Sabor & $7,56 \pm 1,69^{\mathrm{a}}$ & $7,77 \pm 1,25^{\mathrm{a}}$ & $7,80 \pm 1,64^{\mathrm{a}}$ \\
Textura & $7,66 \pm 1,54^{\mathrm{a}}$ & $7,70 \pm 1,30^{\mathrm{a}}$ & $7,77 \pm 1,35^{\mathrm{a}}$ \\
Global & $7,78 \pm 1,30^{\mathrm{a}}$ & $7,87 \pm 1,18^{\mathrm{a}}$ & $7,86 \pm 1,50^{\mathrm{a}}$ \\
Intenção de compra & $3,36 \pm 0,75^{\mathrm{a}}$ & $3,27 \pm 0,77^{\mathrm{a}}$ & $3,26 \pm 0,86^{\mathrm{a}}$ \\
\hline
\end{tabular}

*Letras idênticas em uma mesma linha indicam resultados estaticamente iguais pelo Teste de Tukey (p < 0,05). Fonte: Autores. 
Em relação ao atributo aparência as formulações não apresentaram diferenças estatísticas. As médias para esse quesito ficaram entre 7 e 8, o que, na escala hedônica, corresponde à "gostei moderadamente" e "gostei muito". Mesmo comportamento foi verificado para o aroma.

Considerando que atualmente boa parte da população desconhece ou possui impressões negativas em relação ao quesito sabor dos produtos considerados funcionais, foi importante perceber que o frozen iogurte padrão não teve diferença significativa em relação a F1 e F2. Em relação ao kiwi, Sandes et al. (2015), associaram noni e outras frutas em frozen, sendo o kiwi o segundo melhor avaliado pelos provadores, perdendo apenas para o morango. Assim como na textura, a adição de FOS não exerceu influência perceptíveis pelos provadores sobre este atributo. Em sobremesas lácteas, o que influencia e favorece o sabor e a textura mais macia e cremosa é a gordura a do leite (Alves et al., 2009).

Apesar dos frutooligossacarídeos terem exercido pouca influência na percepção dos atributos sensoriais do frozen iogurte saborizado com kiwi, Balthazar et al. (2015), ressaltam a possibilidade de produzir sorvetes enriquecidos com prebióticos, garantindo menor intensidade de doçura e atributos funcionais diferenciados, com benefícios para a saúde proporcionados pelo ingrediente prebiótico. Segundo Castro (2014), as formas convencionais de sorvete apresentam elevada concentração de gordura e sacarose, elementos que estão relacionados com a textura, consistência e sabor do produto. Entretanto, as fibras solúveis como o frutooligossacarídeo atuam como agente texturizante, principalmente em alimentos com baixo teor de gordura, mantendo as características do produto. Em relação a intenção de compra as médias equivaleram a "provavelmente compraria", reafirmando a aceitação do produto entre o público que a experimentou.

\section{Considerações Finais}

Foi possível obter frozen iogurte saborizado com kiwi e enriquecido com frutooligossacarídeo, com conteúdo de lipídeos e proteínas dentro da legislação para gelados comestíveis. A umidade, parâmetro que sofreu diferenças significativas, influencia pouco já que a conservação do produto é dada pelas baixas temperaturas. O conteúdo de proteína apesar de estatisticamente diferente, parece estar associado a quantidade de iogurte utilizada durante a formulação.

Os atributos sensoriais analisados para todas as formulações não foram estatisticamente diferentes, o que indica que o percentual de frutooligossacarídeo adicionado não influenciou consideravelmente nos aspectos sensoriais do produto, mas pensando nos benefícios adicionais comprovados por pesquisas pode ser alternativa viável para quem tem interesse na funcionalidade dos produtos.

\section{Agradecimentos}

Ao Instituto Federal de Educação, Ciência e Tecnologia do Rio Grande do Norte - Campus Pau dos Ferros.

\section{Referências}

Abis (2018). Associação Brasileira das Indústrias de Sorvetes. http://www.abis.com.br/.

Abreu, E., Preci, D., Zeni, J., Steffens, C. \& Steffens, J. (2018). Desenvolvimento de Frozen Yogurt de iogurte em pó de leite de ovelha. Revista Ceres, 65 (1), $7-15$.

Al-sherajia, S. H., Ismaila, A., Manap, M. Y., Mustafac, S., Yusofa, M. R. \& Hassan, F. A. (2013). Prebiotics as functional foods: A review. Journal of Functional Foods, 5(1), 1542-1553.

Alves, L. L., Richards, N. S. P. S., Becker, L. V., Andrade, D. F., Milani. L. I. G., Rezer, A. P. S. \& Scipion, G. C. (2009). Aceitação sensorial e caracterização de frozen yogurt de leite de cabra com adição de cultura probiótica e prebiótico. Ciência Rural, 39(9), 2596-2600.

An, R. \& Jiang, N. (2017). Frozen yogurt and ice cream were less healthy than yogurt, and adding toppings reduced their nutrition value: evidence from 19992014 National Health and Nutrition. Nutrition Research, 42(1), 64-70.

Aoac (2010). Association of Official Analytical Chemists: Official Methods of Analysis, USA, (18a ed.), 1094p. 
Araújo, A. L. (2011). Elaboração e aceitação de frozen yogurt sabor frutos do cerrado. (Trabalho de conclusão de curso de Bacharelado em Química Industrial) - Universidade Estadual de Goiás, Anápolis, GO, Brasil.

Balthazar, C. F., Silva, H. L. A., Celeguini, R. M. S., Santos, R., Pastore, G. M., Conte Junior, C. A., Freitas, M. Q., Nogueira, L. C., Silva, M. C. \& Cruz, A. G. (2015). Effect of galactooligosaccharide addition on the physical, optical, and sensory acceptance of vanilla ice cream, Journal of Dairy Science, 98(7), 4266-4272.

Brasil (2000). Agência Nacional de Vigilância Sanitária. Consulta pública n 28, de 01 de junho de 2000. Regulamento Técnico para Fixação de Identidade e Qualidade de Gelados Comestíveis, Preparados, Pós para o Preparo e Bases para Gelados Comestíveis.

Brasil (2008). Agência nacional de Vigilância Sanitária. Lista de alegações de propriedade funcional aprovadas.

Castro, E. A. (2014). Elaboração de frozen de bebida láctea fermentada probiótica sabor cajá-umbu. (Dissertação de Mestrado em Tecnologia de Alimentos) Instituto federal de educação ciência e tecnologia do Ceará, CE, Brasil.

Chen, L. \& Karboune, S. (2019). Prebiotics and health: properties, functionalities, production, and overcoming limitations with second-generation levan-type fructooligosaccharides. Encyclopedia of Food Chemistry, 3, 271-279.

Costa, M. F., Pimentel, T. C., Guimaraes, J. T., Balthazarc, C. F., Rocha, R. S., Cavalcanti, R. N., Esmerino, E. A., Freitas, M. Q., Raices, S. L., Silva, M. C. \& Cruz, A. G. (2019). Impact Of Prebiotics On The Rheological Characteristics And Volatile Compounds Of Greek Yogurt. Lwt, 105, $371-376$.

Dalla, C. F. F. (2008). Desenvolvimento de frozen yogurt com propriedades funcionais. (Dissertação de Mestrado em Ciência e Tecnologia de Alimentos), Universidade Federal de Santa Maria, RS, Brasil.

Dutcosky, S. D. (2013). Análise sensorial de alimentos. (4a ed.), Editora Universitária Champagnat.

Fernandes, R. C. S., Pitombo, V. C., Morais, N. A. R., Salvador, A. C. A., Rezende, L. S., Matias, A. C. G., Furquim, N. R. \& Pereira, I. R. O. (2017). Desenvolvimento e avaliação sensorial de sorvete de iogurte (frozen) funcional com biomassa de banana verde e frutas vermelhas. Revista Uningá Review, $30(2), 17-22$.

Finamac (2019). The Finest Automated Machines. https://www.finamac.com/br/noticias/2012/04/frozen-yogurt-a-sobremesa-que-conquistou-o-brasil.

Folch, J., Lees, M. \& Stanley, G. H. S. (1957). A simple method for the isolation and purification of total lipides from animal tissues. Journal of Biological Chemistry, 226, 497-509.

Goff, H. D. (2011). Ice cream and frozen desserts: product types. In: Encyclopedia of dairy sciences, (2a ed.), Academic Press, London, U K, 2(1), 893-912.

Gomes, A. P. E., Silva, K. E., Radeke, S. M. \& Oshiro, A. M. (2012). Caracterização física e química de kiwi in natura e polpa provenientes da comercialização de Dourados - MS. Revista de Ciências Exatas e da Terra, 1(1), 1-8.

Instituto Adolfo Lutz. (2008). Métodos físico-químicos para análise de alimentos. (4a ed.), 1020.

Ishwarya, S. P. \& Prabhasankar, P. (2013). Fructooligosaccharide - Retention during baking and its influence on biscuit quality. Food Bioscience, 4(1), 68-80.

Isik, U., Boyacioglu, D., Capanoglu, E. \& Erdil, D. N. (2011). Frozen yogurt with added inulin and isomalt. Journal of Dairy Science. 94(1), $1647-1656$.

Juliano, R. S., Augusto, M. M., Zamelli, C. A., Fear, A. C., Pinheiro, A. C. \& Sarkis, S. S. J. (2014). Desenvolvimento de sobremesa láctea tipo frozen yogurt com características funcionais. In: Congresso brasileiro de Engenharia Química, 20. Florianópolis- SC.

Mapa (2005). Ministério da Agricultura, Pecuaria e Abastecimento. Manual de Métodos de Análises de Bebidas e Vinagres - Açucares redutores (Método 14). Instrução Normativa $\mathrm{N}^{\circ} 24$, de 08 de setembro de 2005.

Monteiro, R. C. R., Veloso, C. R., Neres, L. S., Lourenço Júnior, J. B., Pacheco, E. A., Abe Sato, S. T., Santos, M. A. S. Nahum, B. S. \& Ribeiro, I. A. (2015). Desenvolvimento e avaliação da qualidade de sorvete de iogurte simbiótico, de leite de búfala enriquecido com polpa de açaí (Euterpe oleracea). Nucleus, $12(2), 1-8$.

Multon, J. L. (2007). Le sucre, les sucres, les edulcorants et les glucides de charges dans les IAA. In: Silva, A. S. S., Haas, P., Sartori, N. T., Anton, A.A. \& Francisco, A. Frutooligossacarideos: Fibras Alimentares Ativas. B.CEPPA, Curitiba, 25(2), 295-304.

Nogueira, L. M. S., Figueiredo, P. S., Candido, C. J., Miyagusku, L., Campos, R. P., Hiane, P. A., Guimarães, R. C. A. \& Aréco, A. E. T. (2018). Caracterização e aceitação sensorial de frozen yogurt formulado com polpa de laranjinha de pacu (Pouteira glomerata (Miq.) Radlk) e culturas probióticas. Ambiência, 12(1), 174-185.

Passos, L. \& Park, Y. K. (2003). Frutooligossacarídeos: implicações na saúde humana e utilização em alimentos. Ciência Rural, $33(2)$, 385-390.

Pereira, G. G., Rafael, L. M., Gajo, A. A., Ramos, T. M., Pinto, S. M., Abreu, L. R \& Resende, J. V. (2012). Influência do pH nas características físicoquímicas e sensoriais de frozen yogurt de morango. Semina: Ciências Agrárias, 33(2), 675-686.

Ribeiro, M. C. O. (2012) Caracterização do Pediococcus acidilactici b14 quanto às propriedades probióticas e sua associação com Lactobacillus acidophilus atcc 4356 com aplicação em sobremesa com soja aerada potencialmente simbiótica. (Tese em Engenharia de Bioprocessos e Biotecnologia), Universidade Federal do Paraná, Curitiba, Brasil.

Sandes, F. S. A., Pinto, D. M., Cavenaghi, D. F. L. C. \& Duarte, J. M. A. (2015). Elaboração de frozen de noni associado com outras frutas antioxidantes. Revista Eletrônica do Univag, 12, 1-12. 
Research, Society and Development, v. 10, n. 8, e5710817031, 2021

(CC BY 4.0) | ISSN 2525-3409 | DOI: http://dx.doi.org/10.33448/rsd-v10i8.17031

Silva, M. I., Sousa, F. C., Martins, J. N. \& Ribeiro, M. C. M. (2016). Avaliação da qualidade físico-química de kiwi (Actinidia deliciosa A. chevalier cultivar 'hayward') minimamente processado. I Congresso Internacional da Ciências Agrárias, COINTER.

Soquetta, M. B. (2015). Caracterização físico-química, microbiológica e compostos bioativos de farinhas de cascas e bagaço de kiwi (Actinidia deliciosa) e sua aplicação em patê. (Dissertação em Ciência e Tecnologia de Alimentos), Universidade Federal de Santa Maria.

Souza, R. L., Feitosa, B. F., Oliveira, E. N. A. \& Oliveira, S. N. (2017). Elaboração e caracterização de frozen yoghurt sabor tamarindo. Revista Brasileira de Agrotecnologia, 7(1), 22-25.

Statsoft, Inc. (2004). Statistica (data analysis software system), version 7.

Zacarchenco, P. B., Gallina, D. A., Van Dender, A. G. F. \& Moreno, I. (2019). Prebióticos em produtos lácteos. Anuário Leite e Derivados, 36-44. http://www.ital.sp.gov.br/tecnolat/arquivos/artigos/PrebioticosProdutosLacteosRevistaLeiteDerivados.pdf. 